Document downloaded from:

http://hdl.handle.net/10251/155963

This paper must be cited as:

Bessini, J.; Lazaro, C.; Casanova Colón, J.; Monleón Cremades, S. (2019). Efficiency-based design of bending-active tied arches. Engineering Structures. 200:1-11.

https://doi.org/10.1016/j.engstruct.2019.109681

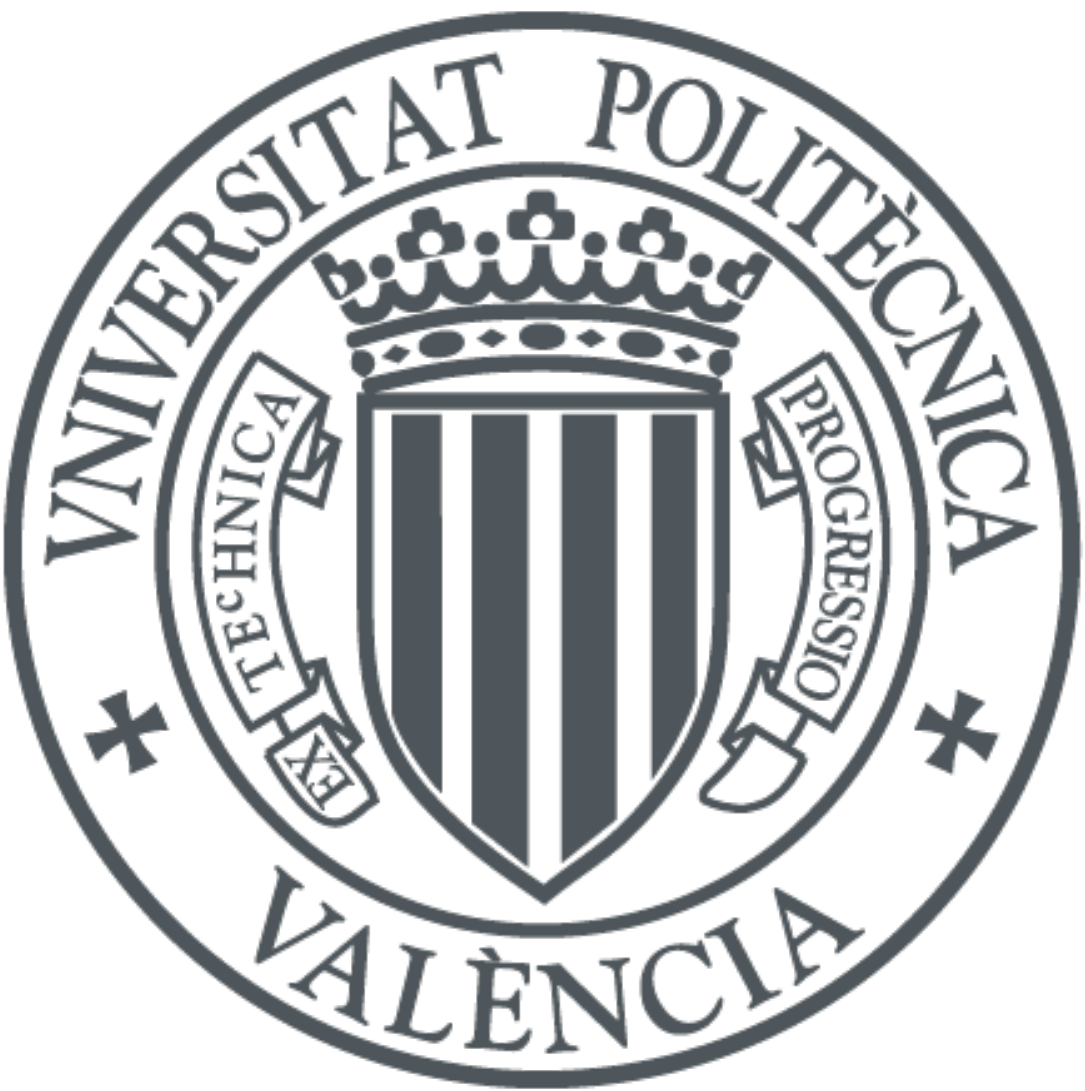

The final publication is available at

https://doi.org/10.1016/j.engstruct.2019.109681

Copyright Elsevier

Additional Information 
This paper must be cited as:

Bessini, J.; Lázaro, C.; Casanova, J.; Monleón, S. (2019). Efficiency-based design of bending-active tied arches. Engineering Structures. Vol. 200, 1 December 2019, 109681.

doi: 10.1016/j.engstruct.2019.109681

The final publication is available at:

https://doi.org/10.1016/j.engstruct.2019.109681

Copyright

Elsevier 
This work is licensed under a Creative Commons "Attribution-NonCommercial-NoDerivatives $4.0 \mathrm{In}-$ ternational"' license.

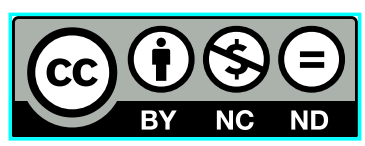




\title{
Efficiency-based design of bending-active tied arches
}

\author{
J. Bessini ${ }^{\mathrm{a}, *}$, C. Lázaro ${ }^{\mathrm{a}}$, J. Casanova ${ }^{\mathrm{a}}$, S. Monleón ${ }^{\mathrm{a}}$ \\ ${ }^{a}$ Departamento de Mecánica de Medios Continuos y Teoría de Estructuras, Universitat \\ Politècnica de València, Camino de Vera s/n, 46022 Valencia, Spain
}

\begin{abstract}
Active bending is recently attracting considerable attention as a new paradigm to build lightweight structures both in research and practice. While there are many references dealing with form-finding methods for bending-active structures, the literature on their performance in relation to their shape and member proportioning is still scarce. This paper addresses the relationship between configuration finding and structural performance in bending-active tied arches: planar arches composed of a bent (active) rod, lower spanning cables and secondary struts that are joined to the rod and act as cable deviators. This simple bending-active arrangement allows to state key relationships between shape, proportion and performance. Starting from the fact that rod segments between struts behave as elastica segments, and selecting the mechanical slenderness of the rod as key parameter, scale-independent relationships between rise-tospan ratio, rod slenderness and stresses after activation have been established for a three-strut tied arch. The limitations posed by keeping stresses in cables after the activation within an acceptable range have been also addressed. Span-deflection ratios corresponding to Eurocode loads for the serviceability limit state have been obtained for a set of three-strut configurations using a non-linear structural model. Results have been represented in terms of rod slenderness, cable slenderness and rise-to-span ratio. The same procedure has been used to determine and represent proper utilization ratios for rod cross-
\end{abstract}

\footnotetext{
${ }^{*}$ Corresponding author

Email address: juabesmu@mes.upv.es (J. Bessini )
}

Preprint submitted to Engineering Structures

December 19, 2019 
sections in ultimate limit state. All the results have been combined to show the design space corresponding to the given constraints and to exemplify how to extract from it a suitable structural configuration. Finally, we explain how to extend the proposed method to design bending-active tied arches with an arbitrary number and proportion of deviators.

Keywords: active bending, elastica, tied arch

\section{Introduction}

Bending-active structures constitute a structural type in which certain flexible structural members are initially bent into curved shapes and then stabilized to form a complete structural system. Because of the geometrical and mechan-

5 ical properties of the bent (or active) members, they are in general ligthweight structures that achieve the desired stiffness due to the curved shape acquired by active elements and to the action of stabilizing additional members [1]. A pioneering architectural realization using the bending active principle is the Mannheim Multihalle (1976) [2] designed by Frei Otto and conceived as a freeform grid of bent timber members; advantage is taken of the flexibility of the timber lathes during the erection process. Otto's concept meant the birth of a specific structural type: the elastic gridshell; a surface structure in which a flat quadrangular mesh of initially straight members with rotation-free joints is lifted up into the desired configuration and stabilized by means of additional structural members.

The interest in bending-active structures has experienced considerable growth in the last years, both in research and in realizations. A number of examples of bending-active structures, as pre-bent composite beams [3], gridshells made of composite materials [4, 5] or timber gridshells [6], have an experimental nature; 20 others -e.g. Faraday Pavilion [7, ICD/ITKE Research Pavillion [8], Ongreening Pavillion [9]- are of ephimeral and/or sculptural nature.

As in the case of tensile structures, the design of bending-active structures requires an initial form-finding step to determine the initial geometry of the 
system. Comparing, for example, a gridshell with a cable net, there is additional complexity in the gridshell due to the fact that member bending will also involve shear and possibly axial forces, and all these will be present in the interaction between members once the equilibrium configuration is reached. In addition to the pure form-finding problem, bending-active structures require tracking the full deformation path of the structural elements from an initial unstressed configuration because the target configuration can be considered a post-buckling state of the initial state. Once the target shape has been reached and stabilized, the structural behaviour is considerably influenced by the inherited stress state.

The first proposal to handle the form-finding problem of gridshells was introduced by Otto et al. in 10; they studied the uniform mesh net with square 35 cells, explained how to build meaningful physical models for hanging nets and proposed the so-called compass method: a geometric method to find Chebyshev meshes from a given curved shape. Different authors have recently developed tools [1], 12, 13] or proposed extensions [14 for this method which is still used. As starting point, the use of physical models or geometric form-finding techniques provide admissible geometrical approximations, however, finite element models are required to simulate the induced pre-stress state. Therefore, the development of computational form-finding methods has been necessary to advance in this research field. Among different strategies, numerical tools based on explicit methods as dynamic relaxation (DR) with differ45 ent underlying mechanical models with 3 to 6 DoFs are the main trend today 15, 16, 17, 18, 19, 20, 21, 22, 23, 24. Alternatively, the availibity of FEM packages has also led to form-finding procedures based on finite element models, for example, Lienhard proposed a form-finding method where unstressed members reach their target shape by shortening non-linear virtual links [25]. Latest trends rely on the use of isogeometric finite elements implemented into a CAD environment [26, 27.

As it can be seen in the literature, most attention has been directed to solve the form-finding problem. In contrast, the number of investigations focused on the assessment of the structural performance and efficiency of bending-active 
structures is limited. Lienhard [28] studied the efficiency of several canonical cases of bending-active structures subject to simple loading patterns over a limited number of cases. Douthe [29] considered strength and stiffness criteria to assesss the applicability of different materials for active grid-shell members. The authors [30] analyzed the response of circular and elastica-shaped active arches subject to a point load, and quantified the relation between geometric stiffness, tangent stiffness and the angle at arch ends for different values of the slenderness. More recently, we presented 31] a preliminary study of the relationship between structural shape, activation forces and activation stress levels for bending-active structures considering different structural configurations and loading conditions.

65 This contribution aims to extend the scope of our previous works to assess the relationship between shape and structural performance of canonical planar bending-active arches.

During the conceptual phase, the designer needs to find a compromise between member strength and magnitude of the pre-deformation: to achieve a suitable shape, significant member curvatures are often needed; therefore, slender members are required for stresses to be lower than a certain threshold value in the target configuration. However, too slender members may be prone to local buckling and may render a too flexible structure.

In the design of gridshell-like structures, the structural configuration is typ75 ically targeted to obtain dome shapes, for which wind and snow loads are the most determinant actions over the structure. However, in the case of footbridges, they must withstand heavier service loads; therefore, reaching a tradeoff between member's strength and curvature may be not evident.

In this paper, we study simple planar structures composed of a continuous flexible member that is activated by the action of main cables pulling at both ends of the rod, and secondary struts that deviate the main cable and act at certain cross-sections of the rod. We will use the term bending-active tied arch to refer to them (Fig. 1).

The interest of simple tied arch systems lies in their applicability as individual structural modules to design lightweight footbridges [32] or roofing appli- 


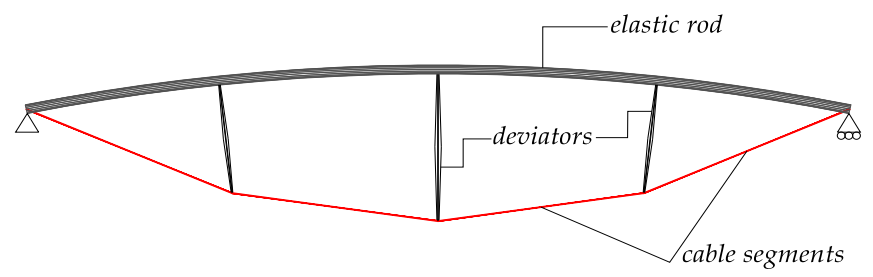

Figure 1: Bending-active tied arch

cations [33, these ones favored by moderate loads and less strict serviceability conditions. The authors have devised a $5 \mathrm{~m}$ span prototype of an experimental lightweight footbridge based on this structural scheme (Fig. 2). The system is composed of a pair of planar bending-active tied arches that are independently activated and connected by hinged links at the level of the main cable and horizontal struts at the level of the rods [34].

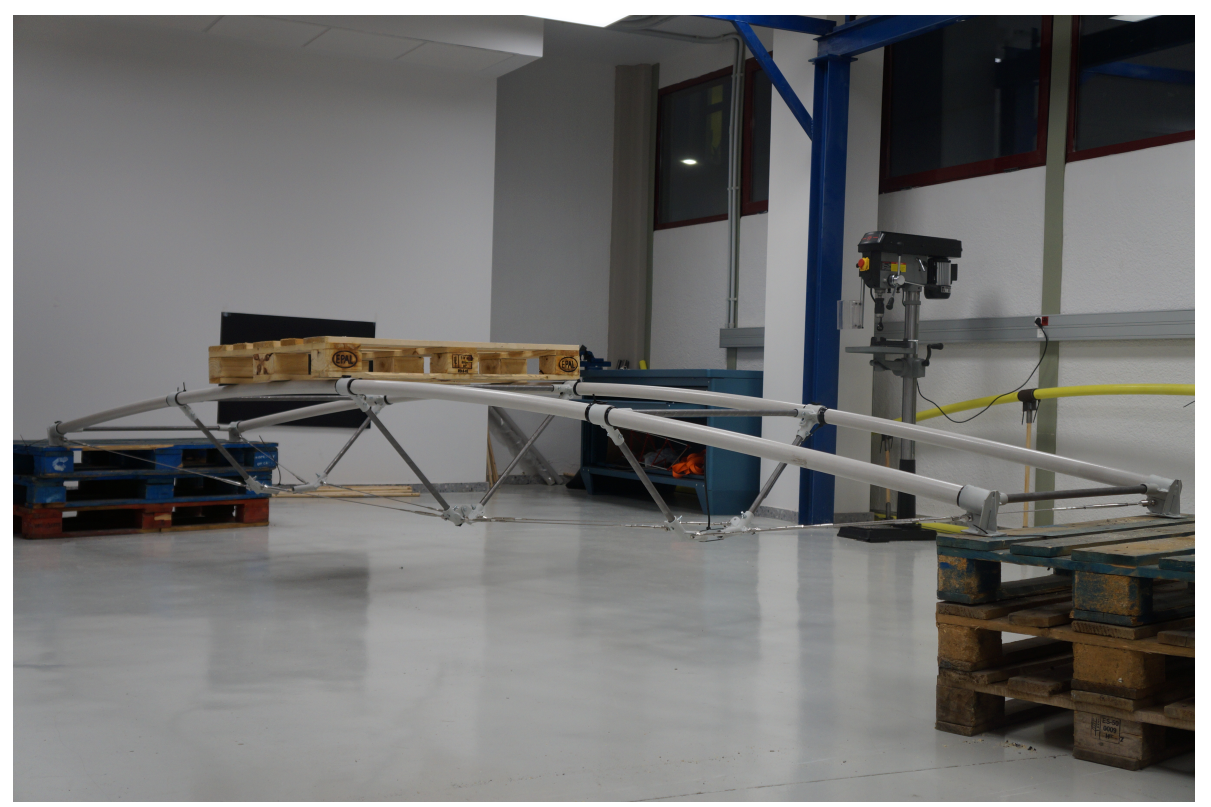

Figure 2: Experimental lightweight footbridge based on the active bending principle

The results that we have obtained regarding the performance of simple tied arch systems for pedestrian footbridge applications show that the limits of solu- 
tions for Eurocode footbridge loads are dictated by (a) instability in the active members, (b) maximum stress after activation and (c) minimum stresses in the activation cables and (d) maximum allowable deflections. The resulting design space is rather narrow, mainly because of the magnitude of the load and the severe limitations for deflections in serviceability limit states posed by codes.

Additionally to these limitations, aspects such as the durability (creep, relaxation or fatigue) or the dynamic behaviour cannot be neglected in the design of bending-active structures, especially in those made of composite materials [5]. In GFRP footbridges it has been observed that the low weight and high flexibility of the structure have a large influence in the dynamic response of the structure, since natural frequencies are expected low and may be activated by pedestrians walking [32]. To complete the study presented in this paper, authors are conducting a research based on experimental tests targeted to evaluate the dynamic behaviour of this kind of structures.

A key observation in this structural system is that the activated member is composed of individual segments between deviators. Each segment of the rod behaves as a segment of an inflexional elastica, whose scale is determined by the ratio between bending stiffness and compressive force [35]. A second key observation is that to each elastica segment corresponds a cable segment, and the (theoretical) axis of the cable segment joins the ideal inflexions of the corresponding elastica segment (Fig. 3). Thereby, once a configuration is found, the size of the entire structure can be adjusted by choosing the required flexural stiffness and the magnitude of forces for each segment of the rod.

The assumption that each segment of the rod behaves as an elastica segment, allows us to make use of the exact solution of the elastica to evaluate the bending effect as well as the influence of the choice of the cross-section and material properties on the initial stress level. As a result of the study, we have obtained a direct relation between shape and activation forces for a given material and cross-sectional shape in terms of non-dimensional magnitudes, which makes it applicable to any size of structure and cross-section properties.

In view of the above finding, it is to be expected that bending-active tied 


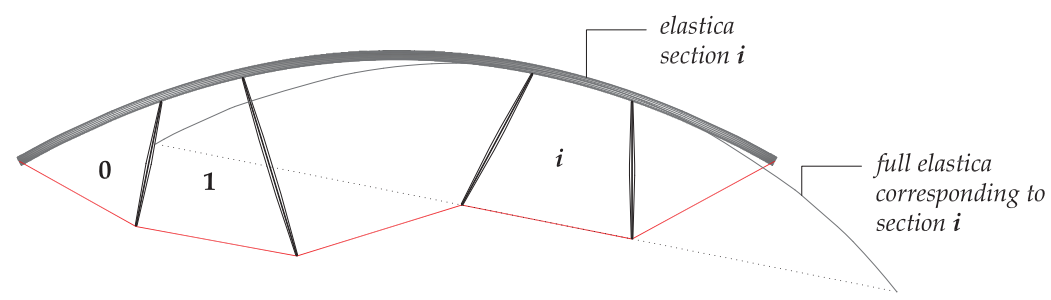

Figure 3: Full (notional) elastica corresponding to segment $i$

125

se generalized for flexible members of any length and stiffness.

Thereby, it is possible to find patterns of relationship between structural shape, activation stress levels, and limits of utilization for bending-active tied arches with a limited number of computer simulations. For that purpose, we use a non-linear finite element commercial software. Starting from a fixed length and given cross-sections, introducing prestressing forces in cables, different structural configurations that keep deviators perperpendicular to the rod are created. From these results, we have obtained stress levels after the activation of the structure as functions of the rise-to-span ratio and the slenderness of the active member. Finally, further numerical analysis has led to establish relations between shape, length and slenderness associated to the serviceability limit state and the ultimate limit state of the structure.

\subsection{Outline of the paper}

This work constitutes a considerable extension of the previous contribution by the authors [31]. The outline of the paper is as follows: In Section 2, the problem of the inflexional elastica is briefly reviewed; using these equations, an analytical expression to evaluate the self-stress after activation in elastica semi-waves is detailed. The correspondence between the structural behaviour 
elastica curve is proved in section 3. In section 4 we introduce the concept of bending-active tied arch and numerical experiments are carry out to evaluate the structural performance due to activation forces and load models. In section 5 we show as example the design of a particular bending-active tied arch using to tied arches with an arbitrary number $n$ of deviators is described in section 6

\section{Stress levels after activation in elastica semi-waves}

The simulation of the activation process of bending-active structures is of crucial importance for their design. Due to the non-linearity of the structural response, it is often not possible to predefine in advance the equilibrium configuration and computational form-finding methods are required for modelling the bending effect. However, in the case of bending-active tied arches, the fact that the rod segments between deviators behave as elastica segments, enables the use of closed-form expressions to evaluate the stress level due to activation forces.

In this section, we propose an analytical expression, based on the equations of the elastica, to quantify the utilization of the material due to the form-finding process. Moreover, we examine the influence of the inteverning variables, such as the slenderness of the rod, the shape of the cross-section or the selected material.

In order to simplify the description, we restrict the analysis to the fundamental aspects of the elastica problem. The full development of this theory can be found in the classical work by Love [36].

\subsection{The fundamentals of the elastica}

The problem of planar bending of an initially straight, non-extensible and non-shear deformable rod subject to compressive forces at its ends is known as 


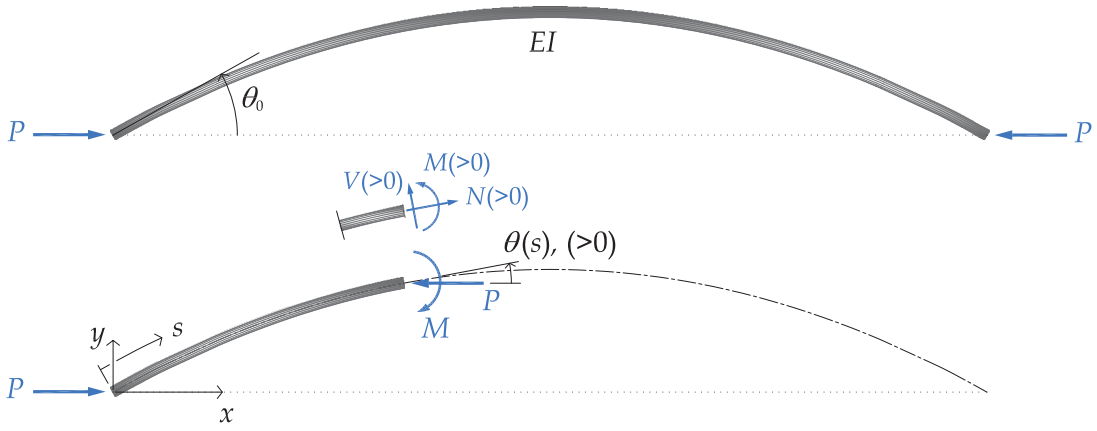

Figure 4: Inflexional elastica

the elastica problem. It was first studied by Euler, based on Bernoulli's assumption of proportionality between flexural moments $M$ and centreline curvatures $\kappa$ at each cross-section.

The constitutive equation for the elastica is $M=E I \kappa$ where the curvature is expressed as $\kappa=d \theta / d s, d s$ is an arc-length parameter, $\theta$ is the cross-section rotation and $E I$ the flexural stiffness. The analytical solution for the arc-length is

$$
s=\frac{1}{2} \sqrt{\frac{E I}{P}} \int_{-\theta_{0}}^{\theta} \frac{1}{\sqrt{\sin ^{2} \frac{\theta_{0}}{2}-\sin ^{2} \frac{\theta}{2}}} d \theta
$$

The applicability of this expression is bounded into the interval $-\theta_{0} \leq \theta \leq \theta_{0}$, where $\theta_{0}$ is the cross-section rotation at the inflexion; therefore the solution cannot be obtained for rotations over $\theta_{0}$. In order to handle this issue, Love [36] introduced the variable $\omega$, defined by

$$
\omega=\frac{\sin \frac{\theta}{2}}{\sin \frac{\theta_{0}}{2}}
$$

substituting in 1 the expression for the arc-length reads as follows

$$
s=\sqrt{\frac{E I}{P}} \int_{-\pi / 2}^{\omega} \frac{1}{\sqrt{1-k^{2} \sin ^{2} \omega}} d \omega
$$


where $k=\sin \frac{\theta_{0}}{2}$ is the reference parameter of the dimensionless solution of the elastica. The integral can reproduce arbitralily long elasticas, because the new variable $\omega$ is not bounded. The solution can be expressed in terms of the incomplete and the complete elliptic integrals of the first kind

$$
\mathrm{F}(\omega, k)=\int_{0}^{\omega} \frac{d w}{\sqrt{1-k^{2} \sin ^{2} w}} \quad \mathrm{~K}(k)=\int_{-\pi / 2}^{0} \frac{d w}{\sqrt{1-k^{2} \sin ^{2} w}}
$$

as

$$
s(\omega, k)=\sqrt{\frac{E I}{P}}(\mathrm{~F}(\omega, k)+\mathrm{K}(k))
$$

\subsubsection{Configuration of the elastica}

Using the incomplete and complete elliptic integrals of the second kind

$$
\mathrm{E}(\omega, k)=\int_{0}^{\omega} \sqrt{1-k^{2} \sin ^{2} w} d w \quad \mathrm{E}(k)=\int_{0}^{\pi / 2} \sqrt{1-k^{2} \sin ^{2} w} d w
$$

the coordinates of the elastica are expressed as follows

$$
\begin{aligned}
& x(\omega, k)=2 \sqrt{\frac{E I}{P}}(\mathrm{E}(\omega, k)+\mathrm{E}(k))-s(\omega, k) \\
& y(\omega, k)=2 \sqrt{\frac{E I}{P}} k \cos \omega
\end{aligned}
$$

\subsubsection{Section forces}

Normal forces and shear forces are obtained as projections of the compressive force, and bending moments as the product of the compressive force times the elastica ordinate.

$$
\begin{aligned}
& N=-P \cos \theta \\
& V=P \sin \theta \\
& M=-P y
\end{aligned}
$$

They can be expressed in terms of the elastica parameters as

$$
\begin{aligned}
& N(\omega, k)=-P\left(1-2 k^{2} \sin ^{2} \omega\right) \\
& V(\omega, k)=-2 P k \sin \omega \sqrt{1-k^{2} \sin ^{2} \omega} \\
& M(\omega, k)=-2 \sqrt{P} \sqrt{E I} k \cos \omega
\end{aligned}
$$




\subsubsection{Scalability of the solution}

We introduce the critical length

$$
l_{c}=\pi \sqrt{\frac{E I}{P}}
$$

defined as the length of a rod with bending stiffness $E I$ for which $P$ is Euler's critical load - this definition was introduced by the authors in 35. Using this definition, the arc-length and the coordinates of the elastica can be expressed as dimensionless quantities

$$
\zeta=s / l_{c} \quad \xi=x / l_{c} \quad \eta=y / l_{c}
$$

Therefore, the non-dimensional arc-length parameter is

$$
\zeta(\omega, k)=\frac{1}{\pi}(\mathrm{F}(\omega, k)+\mathrm{K}(k))
$$

and the non-dimensional coordinates are

$$
\begin{aligned}
\xi(\omega, k) & =\frac{2}{\pi}(\mathrm{E}(\omega, k)+\mathrm{E}(k))-\zeta(\omega, k) \\
\eta(\omega, k) & =\frac{2}{\pi} k \cos \omega
\end{aligned}
$$

These equations show that the shape of an elastica is fully determined by the parameter $k$-or in other words, by the angle at the inflexion (Fig. 5).

On the other hand, the size of the elastica is determined by the critical length $l_{c}$, which acts as a scaling parameter.

Finally, from equation (9) we note that section forces $N, V$ are directly scaled by the compressive force $P$, and bending moments $M$ are scaled by the product of $P$ and the critical length.

To sum up, the shape of the elastica - defined by the parameter $k$ - is fully determined by the angle at the inflexion $\theta_{0}$, and is totally independent from the value of the compressive force $P$ or the bending stiffness $E I$. Once the shape is obtained, the size of the elastica can be scaled by means of the critical length $l_{c}$ (eq. 10). It involves the relation between the bending stiffness $E I$ and the compressive force $P$. 


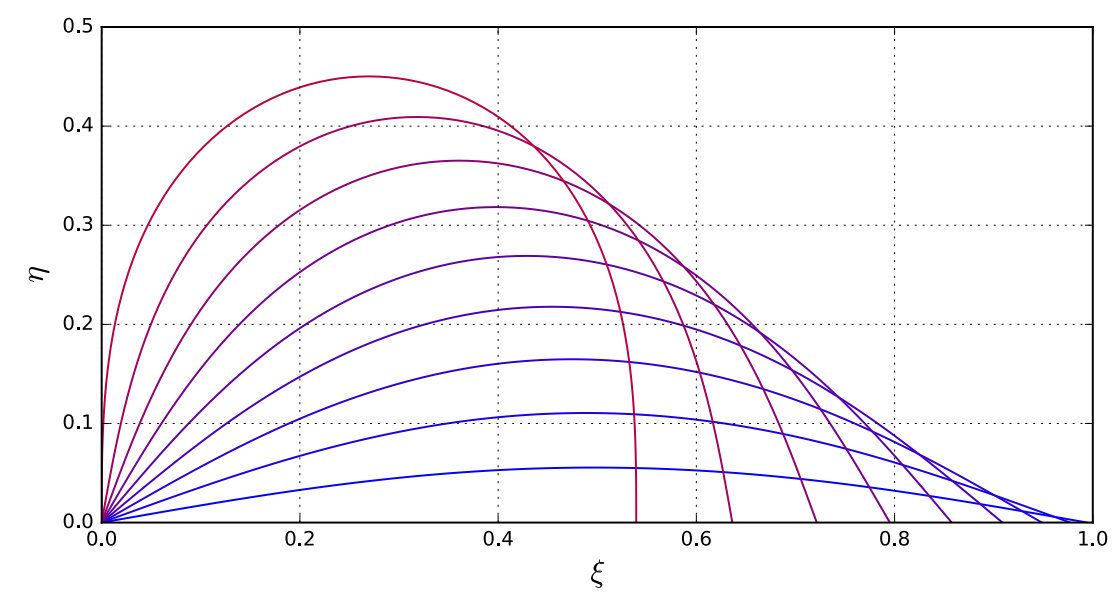

Figure 5: Elastica semi-waves (non-dimensional coordinates) for $\frac{\pi}{18}$ (blue) $\leq \theta_{0} \leq \frac{\pi}{2}$ (red)

For example, once $l_{c}$ has been fixed, the magnitude of the internal forces can be chosen by selecting $P$, and the bending stiffness $E I$ should then be adjusted to be consistent with $l_{c}$.

$$
E I=P\left(\frac{l_{c}}{\pi}\right)^{2}
$$

Alternatively, EI may be prescribed and the magnitud of the forces will be given by

$$
P=\pi^{2} \frac{E I}{l_{c}^{2}}
$$

\subsection{Evaluation of the utilization ratio}

According to the EN 1993-1-1 (Eurocode 3) and as a conservative approximation, the linear summation of the utilization ratio for each stress resultant may be used to verificate the ultimate strength of the cross-section (see equation (6.2) in [37]). This criterium can be written as

$$
\frac{N}{N_{u}}+\frac{M}{M_{u}} \leq 1
$$

where $N$ and $M$ are the section forces produced by bending of a initial straight rod; $N_{u}$ and $M_{u}$ are the design values of the ultimate axial forces and bending 
moments respectively, without considering buckling reduction factors. Substituting (9) in (16) leads to the expression of the utilization ratio in terms of elastica parameters

$$
\frac{P}{f_{u} A} g(\omega, k)+\frac{P l_{c}}{f_{u} W} h(\omega, k)
$$

where $f_{u}$ is the ultimate strength of the selected material, $A$ is the cross-sectional

205 read as follows

We introduce the parameter $\Psi$ as the shape factor of the cross-section: a dimensionless value that characterizes the cross-sectional shape and points out the relationship between the moment of inertia and the cross-sectional area of the active member

$$
\Psi=\frac{I}{A r^{2}}
$$

where $r$ is the distance from the neutral axis to the outermost fibre. Table 1 provides shape factors for typical cross-sections used in the design of bendingactive structures. The shape factor for rectangular cross-sections is equal to $\Psi=0.33$ regardless of the proportion between height and width. For circular solid cross-sections, the shape factor is equal to $\Psi=0.25$. Shape factors for circular hollow cross-sections depend on the thickness of the cross-section; the thinner the thickness, the higher the shape factor. Lienhard [28] points out an optimal ratio $D_{e} / D_{i}=2$ for circular hollow cross-sections, where the stiffness is close to the maximum value and the cross-sectional area is minimized, getting 220 light members easy to manipulate during construction. In addition, the use of a very thin wall increases the risk of local buckling under the effect of external point-loads. Therefore, the most appropriate cross-sectional shape will be the one that avoids local phenomena as crushing or local buckling. 
The parameter $\bar{\lambda}$ is used to characterize the slenderness of the rod. This interpretation is inherited from the definition of mechanical slenderness stated in the Eurocode 3 (see equation (6.5) in [37). It takes into account: the length of a semi-wave of elastica; the shape of the cross-section and the mechanical properties of the material

$$
\bar{\lambda}=\frac{l}{\pi} \sqrt{\frac{A}{I}} \sqrt{\frac{f_{u}}{E}}
$$

Using the definition of slenderness, the utilization ratio (17) can be rearranged as follows

$$
\left(\frac{l}{l_{c}}\right)^{2} \frac{1}{\bar{\lambda}^{2}} g(\omega, k)+\frac{l}{l_{c}} \sqrt{\frac{E}{f_{u}}} \frac{\pi}{\sqrt{\Psi}} \frac{1}{\bar{\lambda}} h(\omega, k)
$$

The quotient $l / l_{c}$ can be stated in terms of the parameter $k$ by means of the non-dimensional arc-length parameter $\zeta(\omega, k)$. Concerning the problem of the inflexional elastica, the length between inflexions is obtained when $\omega=\frac{\pi}{2}$, thereby $\zeta\left(\frac{\pi}{2}, k\right)=\frac{2}{\pi} \mathrm{K}(k)$

$$
\frac{4}{\pi^{2}} \mathrm{~K}(k)^{2} \frac{1}{\bar{\lambda}^{2}} g(\omega, k)+2 \mathrm{~K}(k) \sqrt{\frac{E}{f_{u}}} \frac{1}{\sqrt{\Psi}} \frac{1}{\bar{\lambda}} h(\omega, k)
$$

This expression provides the utilization ratio of any desired cross-section (defined by $\omega$ ) in terms of the slenderness, the material properties and the shape of the cross-section. The non-dimensional expression of the utilization ratio of the cross-section located at midspan -where higher stresses are expected- is obtained by setting $\omega=0$

$$
\frac{4}{\pi^{2}} \mathrm{~K}(k)^{2} \frac{1}{\bar{\lambda}^{2}}+\frac{4 k}{\pi} \mathrm{K}(k) \sqrt{\frac{E}{f_{u}}} \frac{1}{\sqrt{\Psi}} \frac{1}{\bar{\lambda}}
$$

Additionally, a maximum allowable stress can be introduced to limit normal stresses under the form-finding stage. It is estimated that limiting the utilization ratio after activation to $30 \%$ avoids further problems caused by long-term dynamic loading

$$
\frac{4}{\pi^{2}} \mathrm{~K}(k)^{2} \frac{1}{\bar{\lambda}^{2}}+\frac{4 k}{\pi} \mathrm{K}(k) \sqrt{\frac{E}{f_{u}}} \frac{1}{\sqrt{\Psi}} \frac{1}{\bar{\lambda}} \leq 0.3
$$


For example, equation (24) allows to select the minimum slenderness of the

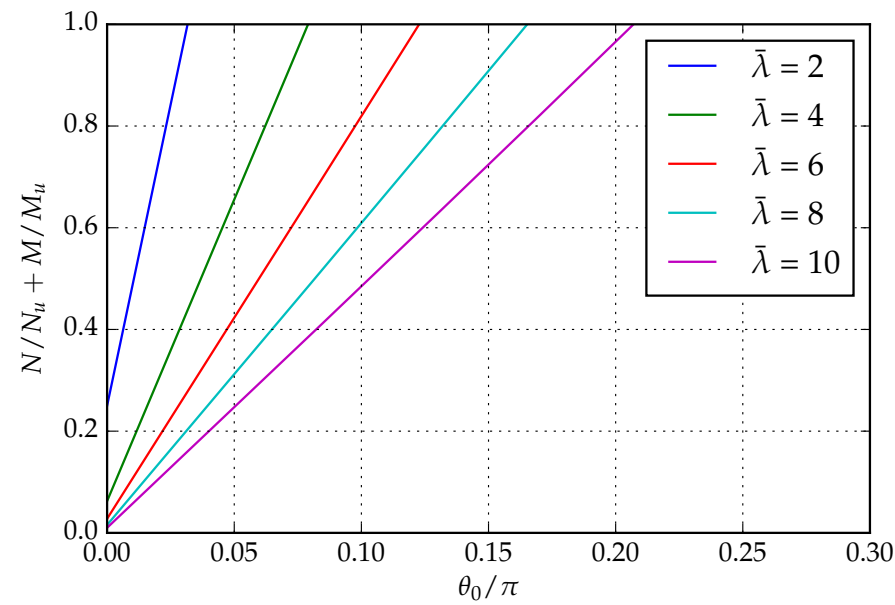

Figure 6: Utilization ratio of elastica semi-waves with rectangular cross-section, angle at the inflexion $\theta_{0}$ and different values of rod slenderness $\bar{\lambda}$

Figure 7 shows how the cross-sectional shape $\Psi$ influences on the utilization ratio of elastica semi-waves, for the same material properties chosen before and for different values of the rod slenderness. According to the diagrams, high shape factors are more advantageous for keeping self-stresses low, however and, as mentioned before, very thin-walled cross-sections may be crushed when applying external loads.

Figure 8 shows the relative weight of the effect of axial forces on the utilization ratio for different rod slenderness. For low values of the cross-section rotation at the inflexion, the effect of axial forces is the most limiting. However, 

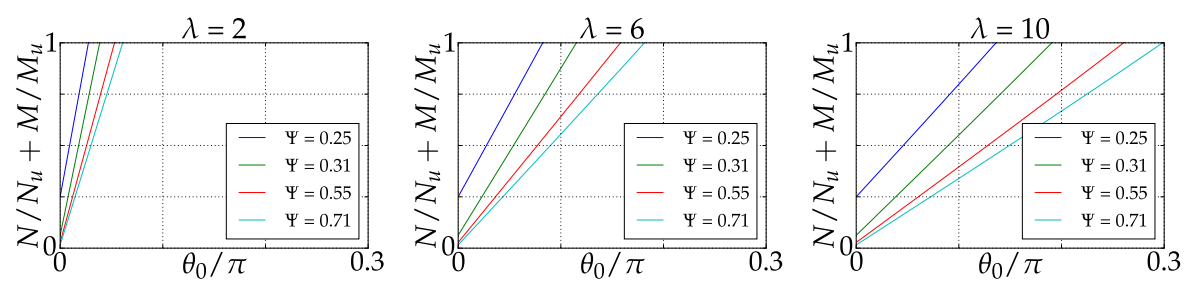

Figure 7: Influence of the shape factor $\Psi$ on the utilization ratio of elastica semi-waves with rectangular cross-section, angle at the inflexion $\theta_{0}$ and different values of rod slenderness $\bar{\lambda}$

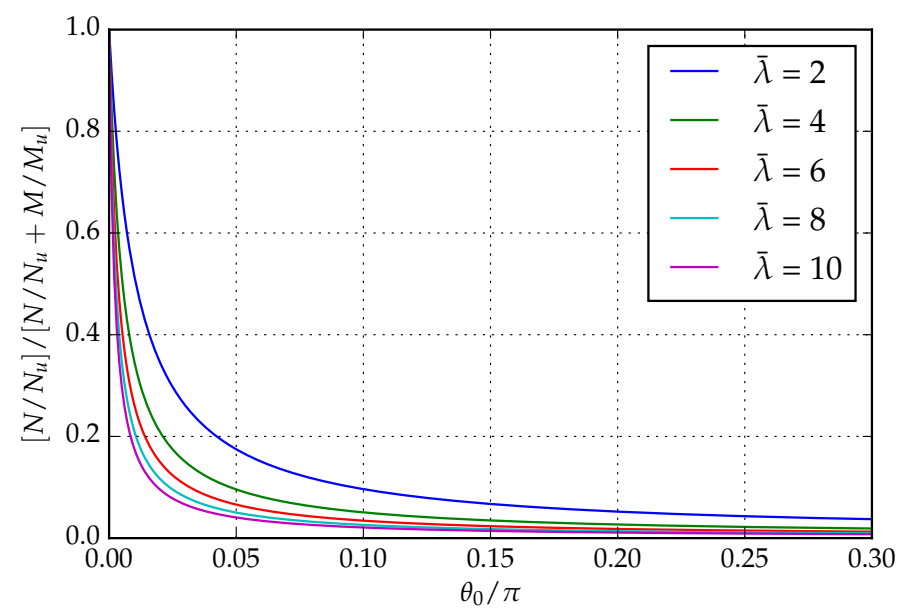

Figure 8: Influence of the term relative to axial forces on the utilization ratio of elastica semi-waves with rectangular cross-section, angle at the inflexion $\theta_{0}$ and different values of rod slenderness $\bar{\lambda}$

as the curvature of the rod increases, stresses produced by bending moments play a more prominent part. As expected, low values of rod slenderness lead to higher utilization ratios due to axial forces. Assuming that the bent rod supports the deck of a footbridge, the maximum gradient of the deck is restricted to $10 \%$ due to functional requirements, which approximately matchs with an angle at the inflexion $\theta_{0}=0.1$. Therefore, the effect of axial forces is low but cannot be fully neglected in the analysis.

The relationship between the ultimate strength $f_{u}$ and the Young's modulus $E$ has been widely used to measure how adequate a material is for bending-active 
structures.

Figure 9 shows the influence of different ratios $f_{u} / E$ on the utilization ratio of elastica semi-waves, with rectangular cross-section and for different values of rod slenderness. 
Ashby [38] represented in form of a diagram this relationship for different

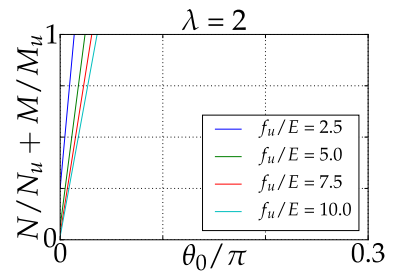
materials; he gathered them by material classes and introduced guidelines to define design regions for certain applications. According to previous works by Lienhard [28] and La Magna [39], suitable materials for bending-active structures have a ratio $f_{u} / E>2.5$ (with $f_{u}$ in $\mathrm{MPa}$ and $E$ in GPa). This requirement is fulfilled by certain types of timber and GFRPs, having the latter ones a ratio $f_{u} / E>10$. As reflected in the diagrams, the use of materials with a high ratio $f_{u} / E$ allows to keep activation stresses low.

Figure 9: Influence of the ratio $f_{u} / E$ on the utilization ratio of elastica semi-waves with rectangular cross-section, angle at the inflexion $\theta_{0}$ and different values of rod slenderness $\bar{\lambda}$

\section{Shape of the rod between deviators after activation}

The assumption that a rod segment between deviators can be modeled as a part of an elastica is one of the keys of this work. It enables the use of closedform expressions (see equations from 21 to 24 to evaluate the stress level at the tensioning stage, and allows to measure how the form-finding parameters influence on the structural configuration. However, this key observation requires some clarifications which are included in the following.

We assume that the rod is non-extensible and non-shear deformable, and we consider the in-plane bending of the rod neglecting self-weight. The first restriction may be adopted without loss of generality for the form-finding problem and will result in a simpler procedure. The non-deformability under shear is a physical consequence of the high slenderness of the rod required for this kind of elements. 

cable force and bending moments acts at ends. At each end, the force $T^{i}$ is decomposed into axial and shear forces, whose value will be distinct, because of the orientation of the centreline is not tangent. These forces at ends, together with the forces of the next segment will be in equilibrium at joining nodes (Fig. ${ }_{280}$ 10). In Fig. 10, the red line is the cable segment that corresponds to the part of the elastica $i$, and the (theoretical) axis that joins the ideal inflexions of the elastica wave (Fig. 3).

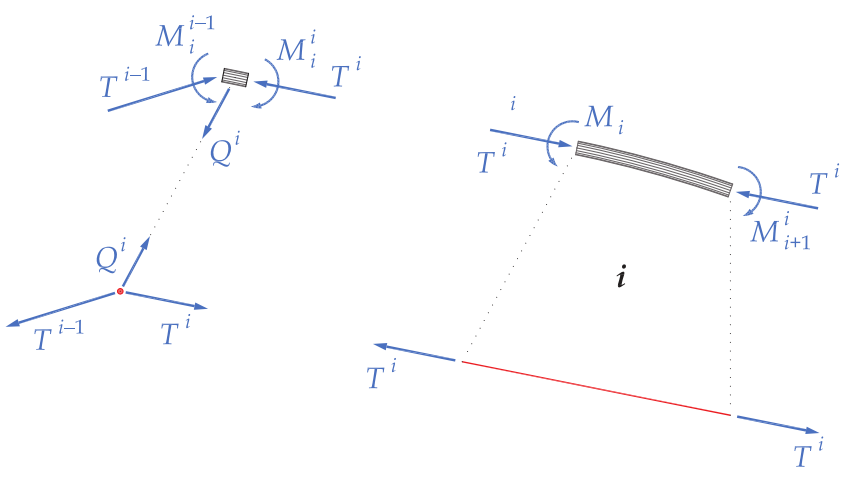

Figure 10: Equilibrium of nodes and elastica segments 
Kirchhoff and Love noted that the equations of in-plane equilibrium of an initially straight, non-extensible and non-shear-deformable rod subject only to forces and/or moments at the end sections are analogue to the equation of movement of a planar pendulum with no restriction on the amplitude of the oscillation. This fact is known as Kirchhoff's kinetic analogy [36]. The corresponding analogues for the inflexional elastica expressed in terms of section forces are

$$
\frac{M^{2}}{2 E I}+N=\mathcal{H}(\text { constant }) \quad N^{2}+V^{2}=P^{2}(\text { constant })
$$

where $\mathcal{H}$ is related with the potential energy density, $P$ is the compressive force and $M, N$ and $V$ are the section forces in the rod.

On the basis of the Kirchhoff's kinetic analogy and using a self-stressed configuration, it is possible to prove that the invariants predicted by the Kirchhoff's kinetic analogy are kept for a segment of rod between deviators.

Figures 11, 12 and 13 show the section forces $(N, M, V)$ obtained in a $\mathrm{FE}$ model. Bending stiffness of the rod equals $E I=23.72 \mathrm{kN} \mathrm{m}$ and the force in cables is equal to $T=50 \mathrm{kN}$. Table 2 shows the values corresponding to the section forces and invariants at different nodes of the rod segment A-E. As can be seen, static invariants remain practically constant along the rod segment (percent errors are below 0.5\%). This error can be attributed to the fact that the software calculation model is not the Euler-Bernoulli beam model.

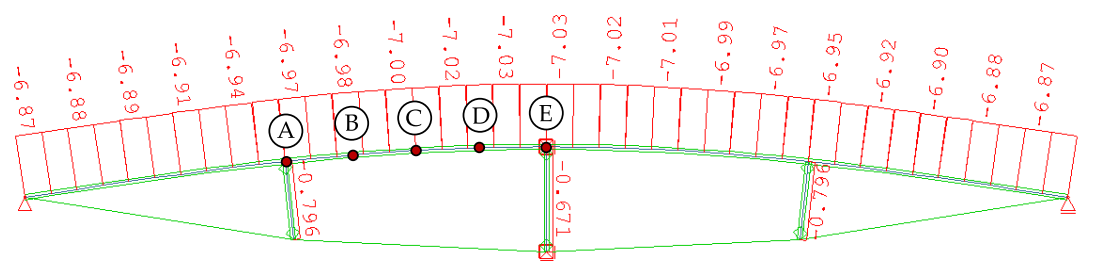

Figure 11: Axial forces $N$ due to activation process 


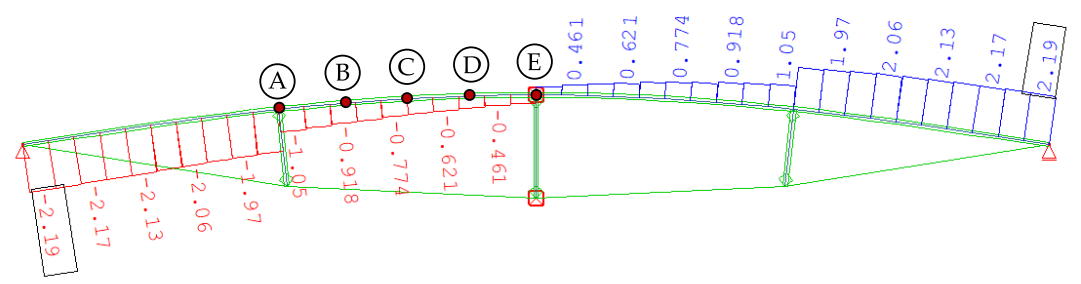

Figure 12: Shear forces $V$ due to activation process

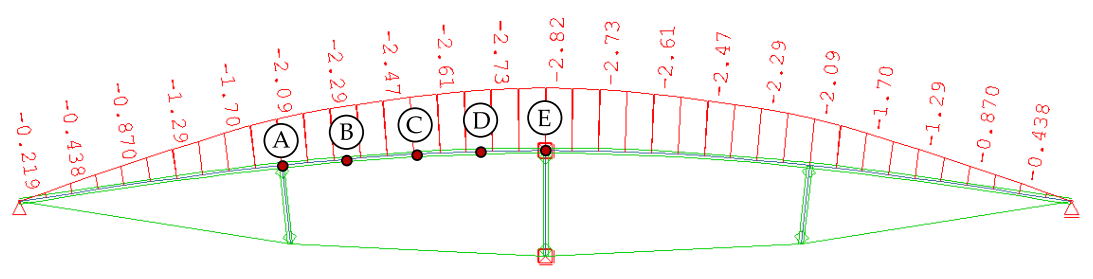

Figure 13: Bending moments $M$ due to activation process

\section{Numerical tests on bending-active tied arches with three deviators}

The term bending-active tied arch refers to a simple planar structure composed of a continuous flexible rod that is activated by cables and deviators, no matter whether they work under compression or under tension. The structural concept is a hybrid between a tied arch and a cable beam and is suited to withstand self-weight and service loads.

During the activation phase, there is a strong interaction between structural proportions, material properties, applied forces and constraints, involving large displacements and rotations of the cross-sections of the rod. Numerical procedures based on non-linear analysis are required in order to simulate the tensioning process, since the activated system cannot be define 'a priori'.

In this section we carry out a set of simulations using specific dimensions of members and materials properties. As rod segments behave as segments of elastica, whose scale is determined by the critical length $l_{c}=\sqrt{E I / T}$, the ratio $l_{c}^{0} / l_{c}^{1}$ between critical lengths of the outer and inner rod segments defines the shape of the structure. Consequently, the shapes and forces obtained for 
a certain definition of members and materials can be scaled by increasing the critical lengths of each segment while keeping their ratio. Thereby, it is possible to generalize the relation between force and shape for flexible members of any length and stiffness basing on particular results.

315 To limit the number of cases and the complexity of the study, we focus on symmetric structures with three equally spaced perpendicular deviators which remain perpendicular to the rod. The following common data have been considered: the upper rod is $4 \mathrm{~m}$ long continuous member with circular hollow cross-section with thickness equal to $10 \%$ of the radius; the deviator lengths are: $h_{q}=0.3 \mathrm{~m}$ at quarters and $h_{m}=0.4 \mathrm{~m}$ at midspan. The ratio between deviator lenghts $h_{q} / h_{m}=0.75$ has been selected after carrying out a parametric study; this ratio provides practically equal axial forces in the deviators after activation and lower deflection under a frequent service load [34]. 


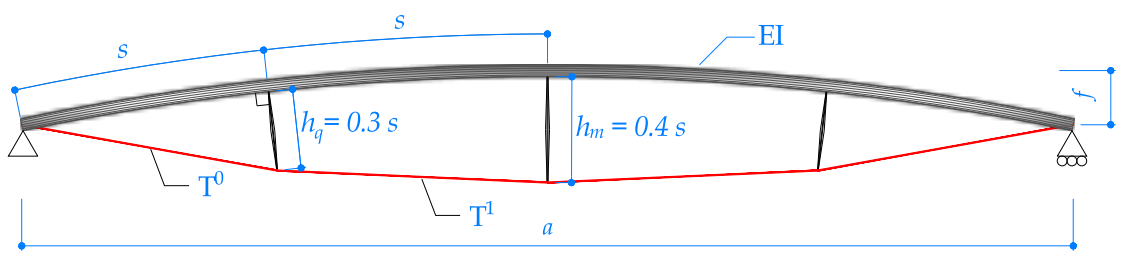

Figure 14: Definition of the input parameters

We have chosen GFRP for the rod (material properties of GFRP are $E=30 \mathrm{GPa}$, $f_{u}=500 \mathrm{MPa}$ ). Cables are not continuous; therefore, cable forces can be different in each cable segment. Steel is used for cables; the material properties of the selected cables are: $E_{s}=110 \mathrm{GPa}, f_{u_{s}}=1570 \mathrm{MPa}$ (Fig. 14).

\subsection{Relationship between shape and activation forces}

In a first step, we generate six different configurations for a bending-active tied arch with three equally spaced and perpendicular deviators. The crosssection of the upper rod is a circular hollow cross-section with $E I=23.72 \mathrm{kN}$ $\mathrm{m}^{2}$. Each self-stressed configuration is obtained introducing a force $T^{0}$ in the outer cable; perpendicularity between rod and deviators is achieved selecting the corresponding force $T^{1}$. Table 3 shows the values of activation forces and the obtained geometric ratios for each structure (Fig. 15). Simulations are carried out using the non-linear FE software SOFiSTiK.

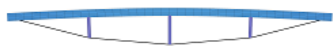

(a)

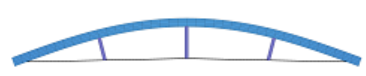

(d)

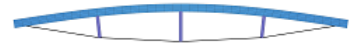

(b)

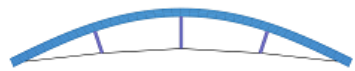

(e)

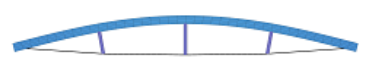

(c)

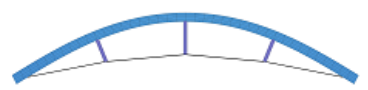

(f)

Figure 15: Different configurations for bending-active tied arches with three equally spaced deviators 


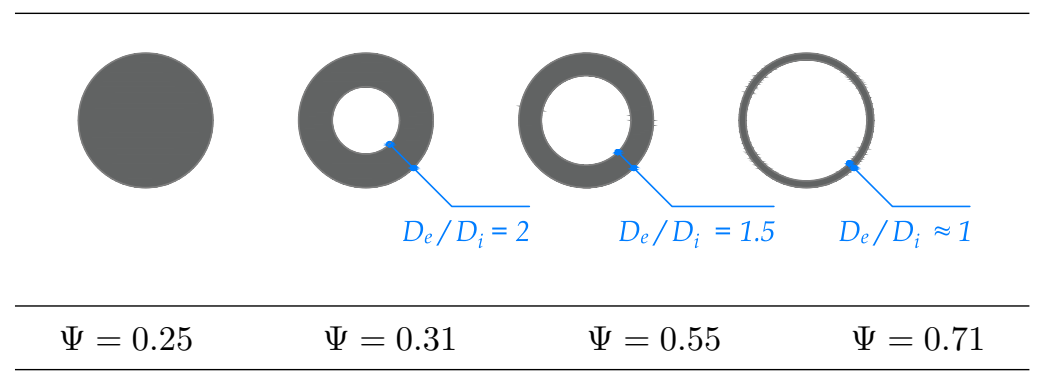

Table 1: Shape factor for circular hollow cross-sections

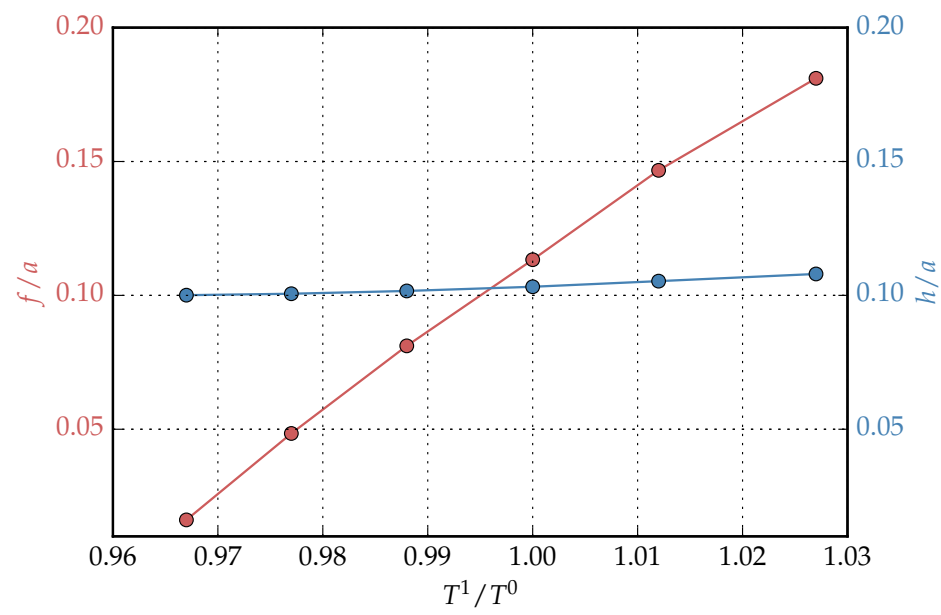

Figure 16: Relation between activation force ratios and non-dimensional shape ratios 


\begin{tabular}{cccccc}
\hline & $N[\mathrm{kN}]$ & $V[\mathrm{kN}]$ & $M[\mathrm{kN} \mathrm{m}]$ & $\mathcal{H}$ & $P[\mathrm{kN}]$ \\
\hline A & -6.97 & 1.05 & 2.09 & -6.88 & 49.68 \\
B & -7.03 & 0.98 & 2.29 & -6.87 & 49.68 \\
C & -7.03 & 0.91 & 2.47 & -6.87 & 49.84 \\
D & -7.02 & 0.77 & 2.73 & -6.87 & 50.2 \\
E & -7.03 & 0.69 & 2.82 & -6.86 & 49.91 \\
\hline
\end{tabular}

Table 2: Section forces and static invariants of the rod segment A-E

\begin{tabular}{rcccccc}
\hline & $\mathrm{a}$ & $\mathrm{b}$ & $\mathrm{c}$ & $\mathrm{d}$ & $\mathrm{e}$ & $\mathrm{f}$ \\
\hline$T^{0}[\mathrm{kN}]$ & 2.475 & 7.211 & 11.678 & 15.892 & 19.890 & 23.629 \\
$T^{1} / T^{0}$ & 0.967 & 0.977 & 0.988 & 1.000 & 1.012 & 1.027 \\
$a[\mathrm{~m}]$ & 3.996 & 3.975 & 3.935 & 3.875 & 3.798 & 3.703 \\
$f / a$ & 0.016 & 0.048 & 0.080 & 0.113 & 0.146 & 0.1811 \\
\hline
\end{tabular}

Table 3: Values of activation forces and resulting geometric ratios for each bending-active tied arch

Figure 16 shows the relation between the non-dimensional shape ratio and the activation force ratio in cables $T^{1} / T^{0}$. Because of the scalability of elastica solutions, these results can be generalized for flexible members of any length and stiffness, as long as deviators are equally spaced and perpendicular to the rod, where the length of central deviator equals $10 \%$ of the length of the rod and the length of lateral deviators equals $75 \%$ of the central deviator.

Using figure 16 and the results in table 3, the configuration associated to a desired shape and size can be easily determined. For example, a $10 \mathrm{~m}$ span and $1.5 \mathrm{~m}$ rise $\operatorname{arch}(f / a=0.15)$ has a force ratio $T^{1} / T^{0}=1.014$. For this $f / a$ ratio, in the original structure, $a=3.770 \mathrm{~m}$ and $T^{0}=20.35 \mathrm{kN}$. The scaling factor for the desired structure will be $10 / 3.77=2.65$; therefore $E I / T^{0}$ should be $2.65^{2}=7.0225$ times larger than in the original one. This can be done with a $2.65 \cdot 4=10.6 \mathrm{~m}$ long rod, using a stronger cross-section, or decreasing the activation force, or a combination of both. 


\subsection{Stress levels in the rod after activation}

The maximum normal stress (in absolute value) acting on the midspan crosssection of the rod after activation has been evaluated for 20 values of rod slenderness $\bar{\lambda}=\frac{s}{\pi} \sqrt{\frac{A}{I}} \sqrt{\frac{f_{u}}{E}}($ from $\bar{\lambda}=0.2$ to $\bar{\lambda}=2.5)$ and 40 values of the rise-to-span ratio (from $f / a=0.01$ to $f / a=0.2$ ), with $s=1 \mathrm{~m}$ (the length of the rod segment between deviators).

The evaluation of the stresses takes into account the axial force and the bending moment produced by the bending effect at midspan, where the curvature reaches its maximum and therefore the bending moment too. In figure 17 we have represented curves corresponding to several ratio of stress-to-ultimatestrength that have been elaborated using the stress values provided by the simulations. Once the shape and size of the structure have been defined, the diagram allows to select the minimum slenderness of the flexural member compatible with a prescribed stress level.

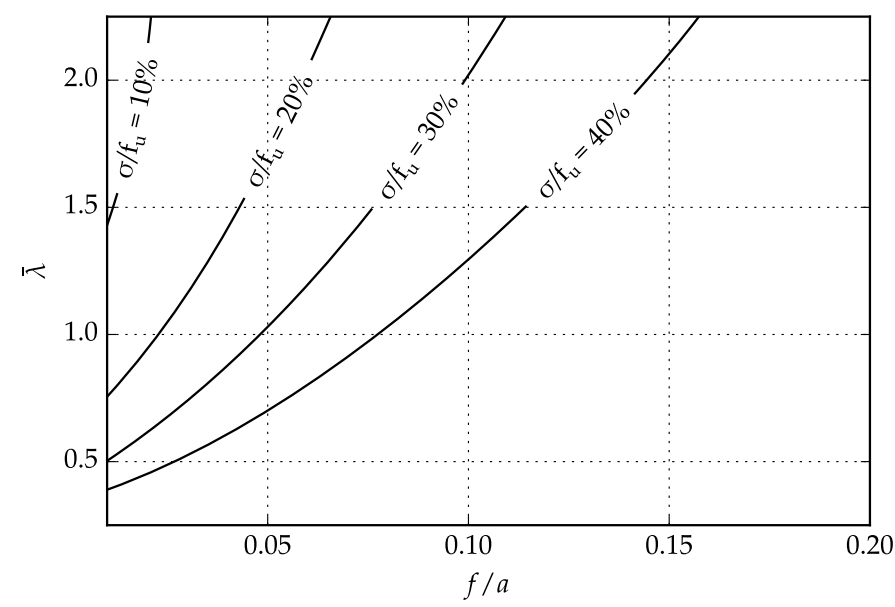

Figure 17: Stress ratio levels after activation in terms of rod slenderness and rise-to-span of the structure 
The selection of the activating cables is crucial in the behaviour of the whole structure. Cables must be carefully designed since oversized cross-sections lead to insufficient cable stresses after the activation stage and undersized crosssections may result in overall excessive flexibility.

Among different commercial typologies of steel cable, we choose a wire rope 7x19+0: 6 individual strands made up of 19 steel wires wrapped around a core made up of the same strand. For the selected wire rope typology, the size of the cable is selected in terms of slenderness. We introduce the following definition of cable slenderness

$$
\overline{\lambda_{c}}=\frac{s}{d} \sqrt{\frac{f_{u_{s}}}{E_{s}}}
$$

where $s$ keeps being the length of the rod segment between deviators and $d$ the external equivalent diameter of the cable.

To evaluate the structural response of the rod and cables in order to satisfy the design limit states, we use the loading value for footbridges defined by the Eurocode [37. For the evaluation of the serviceability limit state, a distributed load corresponding to $40 \%$ of $5 \mathrm{kN} / \mathrm{m}^{2}$ has been applied (frequent value of the service load). The loading pattern consists in a uniform load on a width that we choose to be $10 \%$ of the developed length of the rod (Fig. 18).

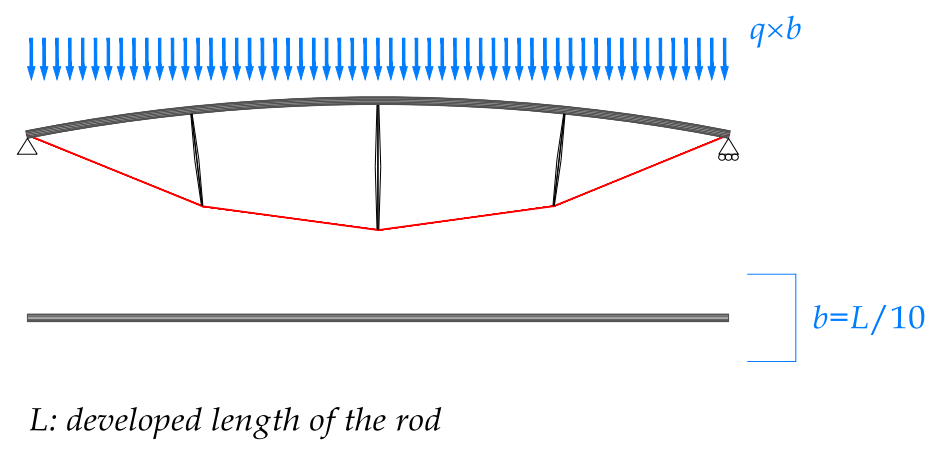

Figure 18: Loading model 
Two series of numerical experiments are carried out: in the first one the cable has been selected so that its slenderness be 15 times the rod slenderness; in the second, the cable slenderness is 5 times the rod slenderness. Within each set, stresses in the rod and cables have been evaluated for 20 values of rod slenderness (from $\bar{\lambda}=0.2$ to $\bar{\lambda}=2.5$ ) and 40 values of the rise-to-span ratio (from $f / a=0.01$ to $f / a=0.2$ ).

Results have been elaborated and summarized in figure 19. It shows the region of the slenderness-shape diagram where for a selected rise-to-span ratio, the diagram allows to choose the cable-rod slenderness ratio compatible to reach at least $10 \%$ of the maximum allowable stress in cables after activation -in order to avoid cable slack- and at most $70 \%$ because of service loads. As can be seen, the choice of the cable-rod slenderness ratio restricts the choice of the rod slenderness for a given rise-to-span ratio: higher cable-rod slenderness ratios allow to select large slenderness rods, which may imply low global stiffness, in contrast, lower cable-rod slenderness ratios lead to oversized rod cross-sections, that are incompatible with keeping activation stresses low.

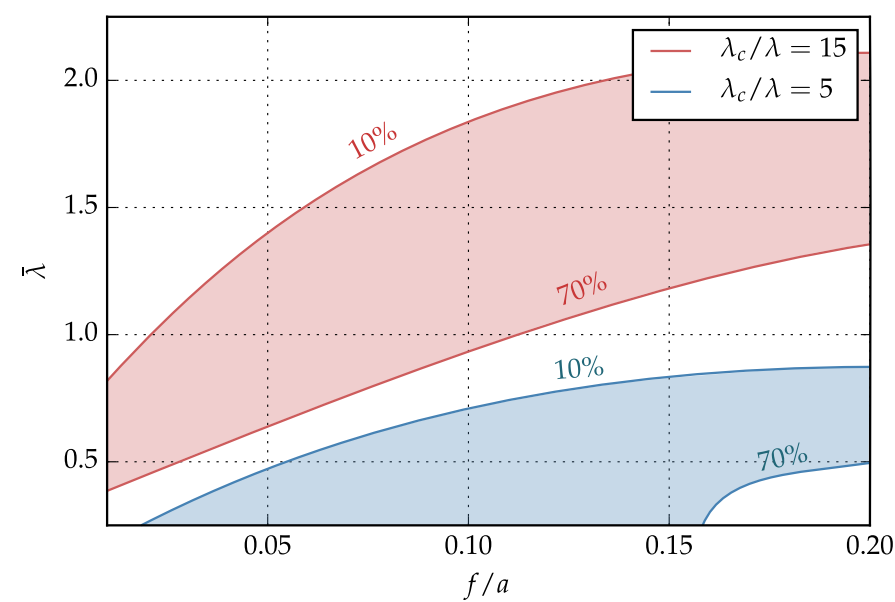

Figure 19: Region where stresses in cables are in the interval between $10 \%$ and $70 \%$ of the maximum allowable stress 


\subsubsection{Performance for serviceability limit state (SLS)}

flections have been evaluated using the same previous set of simulations. Results are summarized in figure 20. Using the diagram, for a selected rod slenderness, the cable can be chosen to achieve a threshold value of deflection. As expected, higher values of rod slenderness lead to larger deflections for a given cable-rod slenderness ratio.

For example, setting a rise-to-span ratio equals $f / a=0.10$ and a threshold value for the deflection equal to $L / 500$-where $L$ is the developed length of the rod-, two configurations are exemplified, each one corresponding to one of the studied cable-to-rod slenderness ratios: a) a rod slenderness $\bar{\lambda}=2$ with a cable slenderness $\bar{\lambda}_{c}=10$ or b) a rod slenderness $\bar{\lambda}=1.15$ with a cable slenderness $\overline{\lambda_{c}}=17.25$. However, solution a) is incompatible with a proper level of stresses in cables.

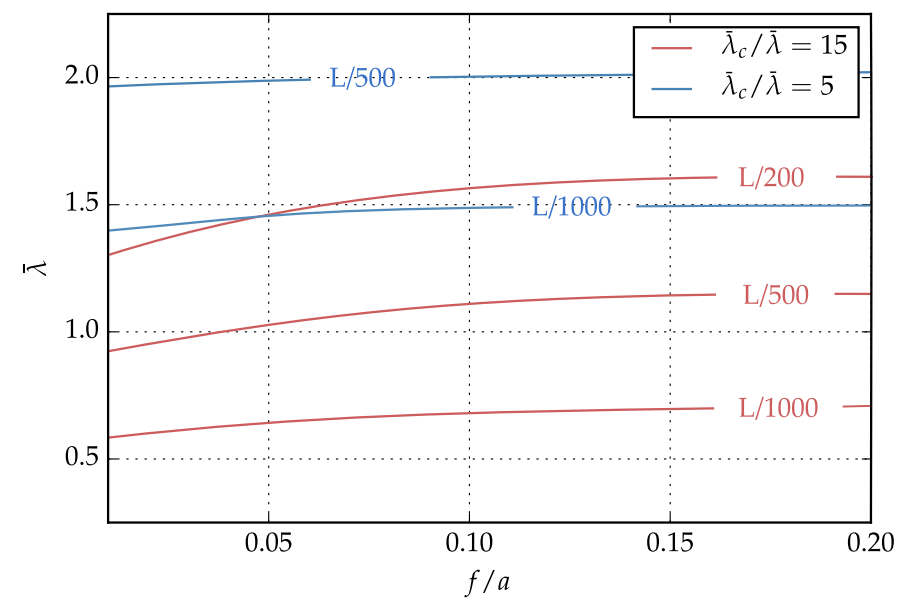

Figure 20: Dimensionless deflections from L/200 to L/1000 for different cable-rod slenderness ratios and a given load in terms of slenderness and shape 
4.4. Performance for ultimate limit state (ULS)

A similar study has been carried out to assess the ultimate limit states, using is consuming a large part of the strength, and therefore the admissible region becomes also more limited. The larger values of the rise-to-span ratio corespond to a slenderness of 1.6 .

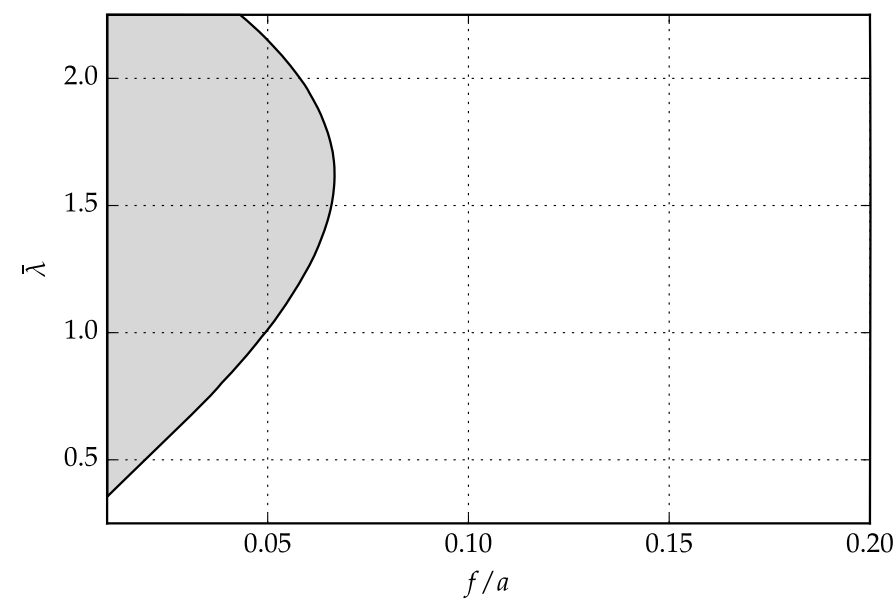

Figure 21: Region where the utilization ratio in the rod is less than 1 for the ultimate limit state 


\section{Example}

In this section we show an example of how the configuration associated to a desired shape and size can be easily determined using the results presented in the previous section.

The design of the bending-active tied arch is based on the following requirements: bending-active rods are designed as $10 \mathrm{~m}$ long continuous GFRP members with thin-walled circular hollow cross-section, the height at midspan should be close to $10 \%$ of the rod length $\left(h_{m}=1 \mathrm{~m}\right)$, the rise-to-span ratio is restricted to $5 \%$, the maximum deflection for frequent loading must be lower than $L / 500$ and the structure has to bear a service load of $5 \mathrm{kN} / \mathrm{m}^{2}$. The mechanical properties of GFRP rod and steel cables correspond to the same setup as in section 4 .

As shown in figure 22, for the selected rise-to-span ratio and choosing the cable slenderness as 15 times the rod slenderness, the maximum rod slenderness should be in the range of 1 . Nonetheless, multiple solutions are possible; any point within the intersecting region (dark red) represents a feasible configuration. It is worth noting that for lower cable-rod slenderness ratios the structure becomes stiffer, but the stress level in the cables restricts considerably the range of solutions; figure 22 also shows the case where selecting a low value for the cable-rod slenderness ratio $\left(\overline{\lambda_{c}} / \bar{\lambda}=5\right)$ may lead to incompatible performances of rod and cables.

Using the definition of slenderness 20, the target configuration is achieved using a circular hollow cross-section with an outer diameter of $190 \mathrm{~mm}$ and a thickness of $9.5 \mathrm{~mm}$. Keeping the established structural proportions, the resulting steel cable is a wire rope $7 \times 19+0$ with a nominal diameter of $20 \mathrm{~mm}$.

\section{Procedure to design bending-active tied arches with $n$ deviators}

In this section we describe a method to design bending-active tied arches with $n$ deviators of any length, based on the results obtained in section 4 


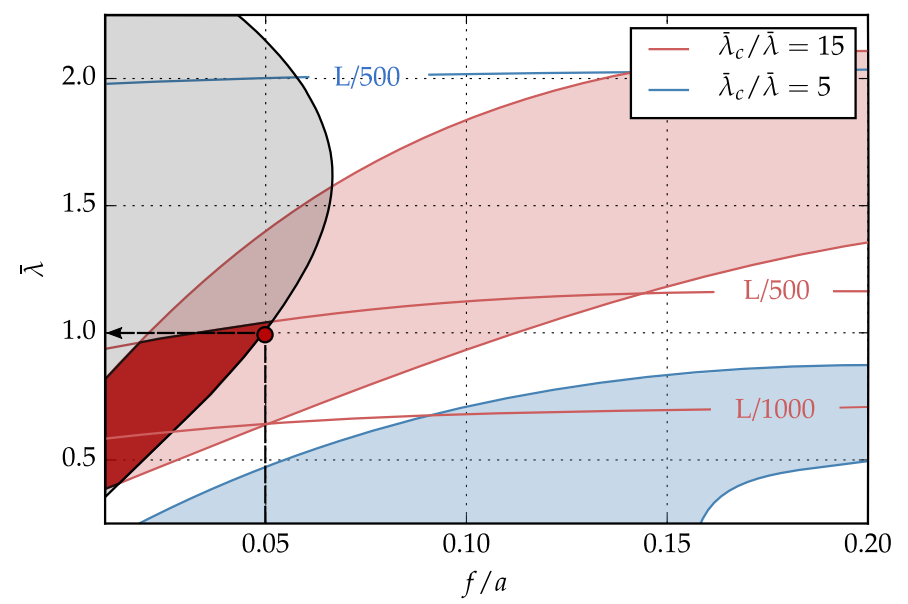

Figure 22: Design diagram for the example

The following procedure is a sequence of orderly steps to reach a feasible solution. As shown in the example in section 5 the problem may have multiple solutions and an iterative calculation would be necessary to reach the best of them. The process is as follows:

1. Define the mechanical properties $E, f_{u}$, for the rod and cables. Deviators can be initially considered as rigid bodies.

2. Define the length of each segment of the rod $s_{i}$, for $i \in\{1 \ldots n\}$, and the lengths of deviators $h_{i}$, for $i \in\{1 \ldots n-1\}$

3. Select the cross-sectional shape $\Psi$ and slenderness $\bar{\lambda}$ for the rod. This choice can be supported by the diagram 17 in a first step.

4. Select the typology and size of steel cables in terms of relative slenderness with the $\operatorname{rod} \overline{\lambda_{c}}=k \cdot \bar{\lambda}$, being $k$ the multiplying factor. It is suggested that $k$ ranges from 10 to 20 .

5. Obtain the configuration associated to a desired shape $f / a$ by means of increasing or decreasing the activation force $T^{0}$. Perpendicularity between rod and deviators can be achieved with a suitable ratio $T^{0} / T^{1}$ (Fig. 16).

6. Check stresses in the $\operatorname{rod} \sigma_{F F, r}$ and cables $\sigma_{F F, c}$ after the activation pro- 
cess. Oversized cables cross-sections can lead to an insufficient stress level (cable slack) at the activation stage and the active member must be slender enough to keep stresses low and limit further problems due to cycling long-term loading. As explained in sections 2.2 and 4.3 , it is suggested that stresses be around $30 \%$ of the material strength in the rod and over $10 \%$ of the cable strength after activation.

7. Simulate the load models and check the serviceability limit state and the ultimate limit state for the rod and cables. Maximum deflections at midspan $v_{(L / 2)}$ and stresses $\sigma_{U L S, r}, \sigma_{U L S, c}$ must be lower than the threshold value posed by codes.

480 Figure 23 shows the process outlined in a flow chart. 
Set initial parameters

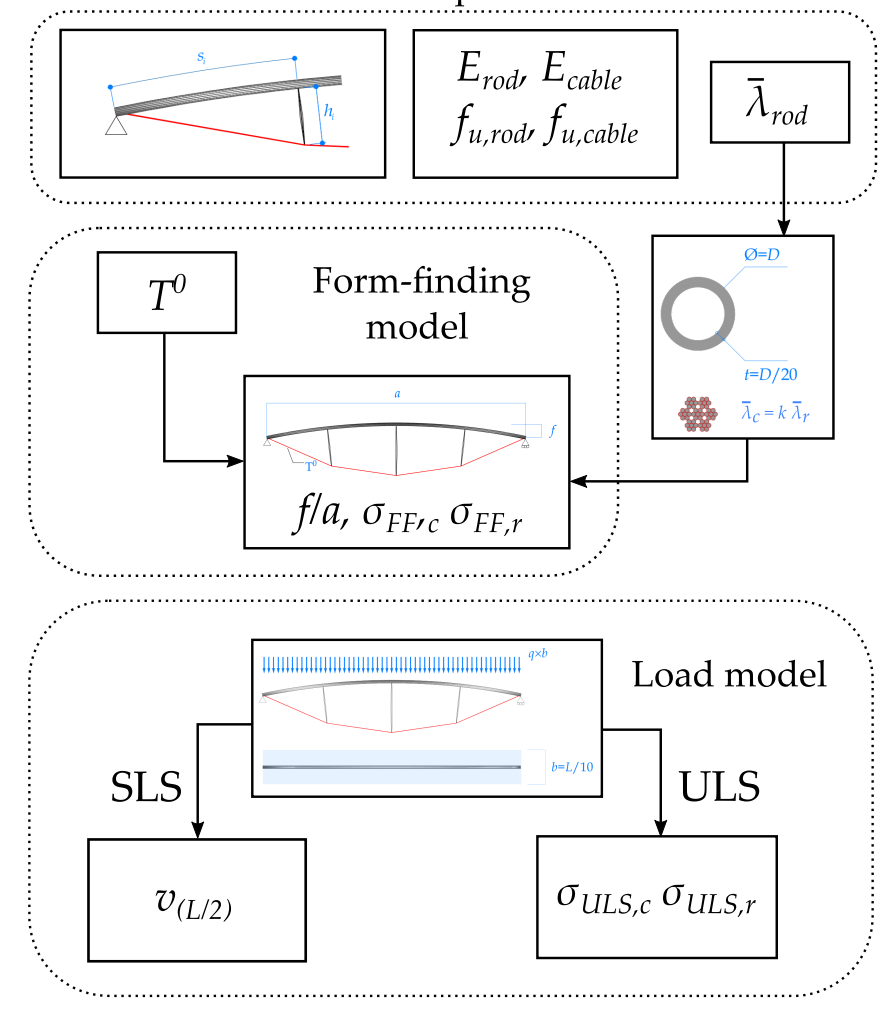

Figure 23: Procedure to design a bending-active tied arch with an arbitrary number of deviators 


\section{Concluding remarks}

The design of bending-active structures is a challenging problem due to the non-linearity of the activation process, the coupling between: cross-section dimensions, structural shape and initial stress state, and the deformability and buckling sensitivity inherent to the resulting lightweight configurations.

In this paper, the activation process and the structural performance under external loads of bending-active tied arches with three perpendicular deviators have been studied by independent reasonings.

First of all, general non-dimensional relations between activation forces and structural shapes have been established in terms of non-dimensional geometric ratios and rod slenderness. The exact solution of the elastica has been used for the evaluation of self-stresses and the influence of the governing variables, after activation in elastica semi-waves (section 2). The paper shows that since the elastica can be scaled as a function of the critical length, and the bending-active tied arch is a sequence of elastica segments, by choosing certain conditions, the whole structure has also scalability properties. Due to the scalability of the solution, the obtained results are applicable to the design of bending-active structures of any size and stiffness. These outcomes have served as a basis to carry out the numerical tests in bending-active tied arches shown in section 4

Secondly, the serviceability limit state and the ultimate limit state have been studied separately by means of two series of numerical experiments using the non-linear FE software SOFiSTiK, the results of which are expressed as a function of the external load. Maximum deflections and stresses in the rod and cables have been evaluated according to the limitations posed by the Eurocode for footbridges. With the outcomes of the study, general non-dimensional diagrams have been obtained for further designs of bending-active tied arches with three deviators of any size and shape. The results show that the design space is limited, mainly because the magnitude of the load and limitations for deflections in serviceability limit states posed by codes are very restrictive. Less restrictive limitations would allow for wider design possibilities. 
Section 5 shows an example that illustrates the way to obtain a feasible configuration compatible to a desired shape and size of a bending-active tied arch with three deviators, for the chosen materials and structural proportions. Finally, a procedure to extend the previous results to tied arches with different 515 number and proportions of deviators has been detailed.

\section{Acknowledgements}

The authors gratefully acknowledge the financial support from the Spanish Ministry of Economy and Competitiveness through grant BIA2015-69330-P (MINECO) and the support from CALTER Ingeniería and SOFiSTiK AG for ${ }_{520}$ providing a software license.

\section{References}

[1] C. Lázaro, J. Bessini, S. Monleón, Mechanical models in computational form finding of bending-active structures, International Journal of Space Structures 33 (2) (2018) 86-97. doi:10.1177/0266351118794277.

[2] E. Happold, W. I. Liddell, Timber lattice roof for the Mannheim Bundesgartensschau, The Structural Engineer 53 (3) (1975) 99-135.

[3] O. Baverel, J. F. Caron, M. Beaugelin, J. Bonthoux, S. Martin, Concept of a beam prestressed by bending: Application to a footbridge in composite materials, Journal of the International Association for Shell and Spatial Structures 51 (3) (2010) 99-106.

[4] C. Douthe, O. Baverel, J. F. Caron, Gridshell in composite materials: Towards wide span shelters, Journal of the International Association for Shell and Spatial Structures 48 (3) (2007) 175-180.

[5] C. Douthe, J. F. Caron, O. Baverel, Gridshell structures in glass fibre 535 reinforced polymers, Construction and Building Materials 24 (9) (2010) 1580-1589. 
[6] S. Pone, S. Colabella, B. Parenti, D. Lancia, A. Fiore, B. D'Amico, F. Portioli, R. Landolfo, M. D'Aniello, C. Ceraldi, Construction and form-finding of a post-formed timber grid-shell, in: Structures and Architecture: Concepts, Applications and Challenges - Proceedings of the 2nd International Conference on Structures and Architecture (ICSA 2013), CRC Press, 2013, pp. 245-252, guimaraes, Portugal, 24-26 July 2013.

[7] P. Nicholas, E. Lafuente-Hernández, C. Gengnagel, The Faraday Pavilion: activating bending in the design and analysis of an elastic gridshell, in: Proceedings of the Symposium on Simulation for Architecture \& Urban Design (SimAUD 2013), Society for Modeling \& Simulation International, 2013, pp. 154-161, san Diego (CA), USA, 7-10 April, 2013.

[8] J. Lienhard, S. Schleicher, J. Knippers, Bending-active structures - research pavilion icd/itke, in: D. Nethercot, S. Pellegrino (Eds.), Proceedings of the IABSE-IASS Symposium - Taller, Longer, Lighter, 2011.

[9] J. Harding, W. Pearson, H. Lewis, S. Melville, The Ongreening Pavilion, in: Advances in Architectural Geometry 2014, Springer International Publishing, 2014, pp. 295-308.

[10] F. Otto, J. Hennicke, K. Matsushita, IL 10 Grid Shells, Institut für Leichte Flächentragwerke, 1974.

[11] L. Bouhaya, O. Baverel, J.-F. Caron, Optimization of gridshell bar orientation using a simplified genetic approach, Structural and Multidisciplinary Optimization 50 (2014) 839-848.

[12] L. Du Peloux, O. Baverel, J.-F. Caron, F. Tayeb, From shape to shell: a design tool to materialize freeform shapes using gridshell structures, in: Design Modeling Symposium, Berlin, Deutschland, 2011.

[13] S. Pone, G. Mirra, E. Pignatelli, D. Lancia, S. Colabella, Specialised algorithms for different project stages in a post-formed timber gridshell design, 
in: Proceedings of the 3rd International Conference on Structures and Architecture, Guimarães, Portugal, 2016.

[14] B. Lefevre, C. Douthe, O. Baverel, Buckling of elastic gridshells, Journal of the International Association for Shell and Spatial Structures 56 (3) (2015) $153-171$.

[15] S. Adriaenssens, M. Barnes, Tensegrity spline beam and grid shell structures, Engineering Structures 23 (1) (2001) 29-36. doi:10.1016/ S0141-0296(00)00019-5

[16] C. Douthe, O. Baverel, J. Caron, Form-finding of a grid shell in composite material, Journal of the International Association for Shell and Spatial Structures 47 (2006) 53-62.

[17] M. R. Barnes, S. Adriaenssens, M. Krupka, A novel torsion/bending element for dynamic relaxation modeling, Computers \& Structures 119 (2013) 60-67. doi:10.1016/j.compstruc.2012.12.027.

[18] B. D'Amico, A. Kermani, H. Zhang, Form finding and structural analysis of actively bent timber grid shells, Engineering Structures 81 (2014) 195-207. doi:10.1016/j.engstruct.2014.09.043.

[19] L. Du Peloux, F. Tayeb, B. Lefevre, O. Baverel, J.-F. Caron, Formulation of a 4-DoF torsion/bending element for the formfinding of elastic gridshells, in: Proceedings of the IASS Symposium 2015, Amsterdam, 2015, pp. 1-15.

[20] G. Senatore, D. Piker, Interactive real-time physics: An intuitive approach to form-finding and structural analysis for design and education, ComputerAided Design 61 (2015) 32-41. doi:10.1016/j.cad.2014.02.007.

[21] B. D'Amico, H. Zhang, A. Kermani, A finite-difference formulation of elastic rod for the design of actively bent structures, Engineering Structures 117 (2016) 518-527. doi:10.1016/j.engstruct.2016.03.034. 
[22] B. Lefevre, F. Tayeb, L. du Peloux, J.-F. Caron, A 4-degree-of-freedom Kirchhoff beam model for the modeling of bending-torsion couplings in active-bending structures, International Journal of Space Structures 32 (2) (2017) 69-83.

[23] J. Bessini, C. Lázaro, S. Monleón, A form-finding method based on the geometrically exact rod model for bending-active structures, Engineering Structures 152 (2017) 549-558.

[24] Y. Sakai, M. Ohsaki, Discrete elastica for shape design of grid-

- shells, Engineering Structures 169 (2018) 55 - 67. doi:https: //doi.org/10.1016/j.engstruct.2018.05.002

600 URL http://wwW.sciencedirect.com/science/article/pii/ S0141029617338695

[25] J. Lienhard, R. La Magna, J. Knippers, Form-finding bending-active structures with temporary ultra-elastic contraction elements, WIT Transactions on the Built Environment 136 (2014) 107-116. doi:10.2495/MAR140091.

[26] A. Bauer, M. Breitenberger, B. Philipp, R. Wüchner, K.-U. Bletzinger, Nonlinear isogeometric spatial Bernoulli beam, Computer Methods in Applied Mechanics and Engineering 303 (2016) 101-127.

[27] P. Längst, A. Bauer, A. Michalski, J. Lienhard, The potentials of isogeometric analysis methods in integrated design processes, in: Proceedings of the IASS Annual Symposium 2017 - Interfaces: architecture.engineering.science, 2017, hamburg, 25-25 September 2017.

[28] J. Lienhard, Bending-active structures : Form-finding strategies using elastic deformation in static and kinetic systems and the structural potentials therein, no. 36 in itke Forschungsberichte, Universität Stuttgart - Institut für Tragkonstruktionen und Konstruktives Entwerfen, 2014.

[29] C. Douthe, Etude de structures élancées précontraintes en matériaux composites : application à la conception des gridshells, Ph.D. thesis, thèse de 
doctorat dirigée par Caron, Jean-François Matériaux et structures Marnela-vallée, ENPC 2007 (2007).

[30] C. Lázaro, S. Monleón, J. Bessini, Tangent stiffness in point-loaded elastica arches, in: Proceedings of the IASS Annual Symposium 2017, Hamburg, 2017.

[31] C. Lázaro, J. Bessini, S. Monleón, Shape and performance of bending-active tied arches, in: Proceedings of the IASS Annual Symposium 2018, Boston, 2018.

[32] J.-F. Caron, S. Julich, O. Baverel, Selfstressed bowstring footbridge in frp, Composite Structures 89 (3) (2009) 489 - 496. doi:https://doi.org/10. $1016 / j$.compstruct.2008.11.009.

[33] K. Noda, Y. Kanebako, Structural design of pre-bent dimensional lumber suspenarches, in: Proceedings of the IASS Annual Symposium 2018, Boston, 2018.

[34] J. Bessini, R. Piñol, C. Lázaro, S. Monleón, Design of an experimental lightweight footbridge based on the active bending principle, in: Proceedings of the IASS Annual Symposium 2018, Boston, 2018.

[35] C. Lázaro, S. Monleón, J. Casanova, Can the force density method be extended for active bending structures?, in: Future Visions - Proceedings of the IASS Symposium 2015, 2015, amsterdam, The Nederlands, 17-20 August 2015.

[36] A. E. H. Love, A Treatise on the Mathematical Theory of Elasticity, 4th Edition, Dover, 1944.

[37] BS EN 1993-1-1 Eurocode 3: Design of steel structures - Part 1-1: General rules and rules for buildings, British Standards Institution, 2005.

[38] M. Ashby, MAterial selection in mechanical design, MRS Bulletin, 2005. 
${ }_{645}[39]$ R. La Magna, Bending-Active Plates :Strategies for the Induction of Curvature through the Means of Elastic Bending of Plate-based Structures., Universität Stuttgart - Institut für Tragkonstruktionen und Konstruktives Entwerfen, 2017. 